Manuscript prepared for Clim. Past

with version 4.2 of the LTEX class copernicus.cls.

Date: 5 February 2014

\title{
Supplementary information to "Nonlinear regime shifts in Holocene Asian monsoon variability: Potential impacts on cultural change and migratory patterns"
}

\author{
J. F. Donges ${ }^{1,2}$, R. V. Donner ${ }^{1,3}$, N. Marwan ${ }^{1}$, S. F. M. Breitenbach ${ }^{4}$, K. Rehfeld ${ }^{1,5}$, and J. Kurths ${ }^{1,6,7}$ \\ ${ }^{1}$ Potsdam Institute for Climate Impact Research, Telegrafenberg A31, 14473 Potsdam, Germany \\ ${ }^{2}$ Stockholm Resilience Centre, Stockholm University, Kräftriket 2B, 11419 Stockholm, Sweden \\ ${ }^{3}$ Department of Biogeochemical Integration, Max Planck Institute for Biogeochemistry, Hans-Knöll-Straße 10, 07745 Jena, \\ Germany \\ ${ }^{4}$ Geological Institute, Department of Earth Sciences, ETH Zurich, 8092 Zurich, Switzerland \\ ${ }^{5}$ Alfred Wegener Institute for Polar and Marine Research, Telegrafenberg A43, 14473 Potsdam, Germany \\ ${ }^{6}$ Department of Physics, Humboldt University, Newtonstr. 15, 12489 Berlin, Germany \\ ${ }^{7}$ Institute for Complex Systems and Mathematical Biology, University of Aberdeen, Aberdeen AB24 3FX, United Kingdom
}

\section{Introduction}

Here, we provide the complete results of applying recurrence network (RN) analysis to the COPRA ensembles corresponding to all records that are considered in our study. This en5 semble analysis allows insights into the effects of dating uncertainties on the proxy records (Fig. S1) as well as the inferred time evolution of indicators of rapid dynamical change (RN average path length $\mathcal{L}$, Fig. S2) and regularity (RN transitivity $\mathcal{T}$, Fig. S3) of monsoonal strength variations. These results are derived applying the methodology described in Section 2 (main text) and can be interpreted along the lines of the discussion in Section 4.1 (main text). Note, that the summary statistics displayed in Fig. 10 (main text) is derived from the results presented in Figs. S2, S3 using the procedure 15 introduced in Section 4.2 (main text).

\section{References}

Breitenbach, S. F. M., Rehfeld, K., Goswami, B., Baldini, J. U. L., Ridley, H. E., Kennett, D., Prufer, K., Aquino, V. V., Asmerom, Y., Polyak, V. J., Cheng, H., Kurths, J., and Marwan, N.: COnstructing Proxy-Record Age models (COPRA), Climate of the Past, 8, 1765-1779, doi:10.5194/cp-8-1765-2012, 2012.

Donges, J. F., Donner, R. V., Rehfeld, K., Marwan, N., Trauth, M. H., and Kurths, J.: Identification of dynamical transitions in marine palaeoclimate records by recurrence network analysis, Nonlinear Proc. Geophys., 18, 545-562, doi:10.5194/ npg-18-545-2011, 2011a.

Donges, J. F., Donner, R. V., Trauth, M. H., Marwan, N., Schellnhuber, H. J., and Kurths, J.: Nonlinear detection of paleoclimatevariability transitions possibly related to human evolution, Proc. Natl. Acad. Sci. USA, 108, $20422-20427$, doi:10.1073/pnas. 1117052108, 2011b.

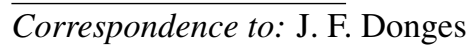

(donges@pik-potsdam.de) 


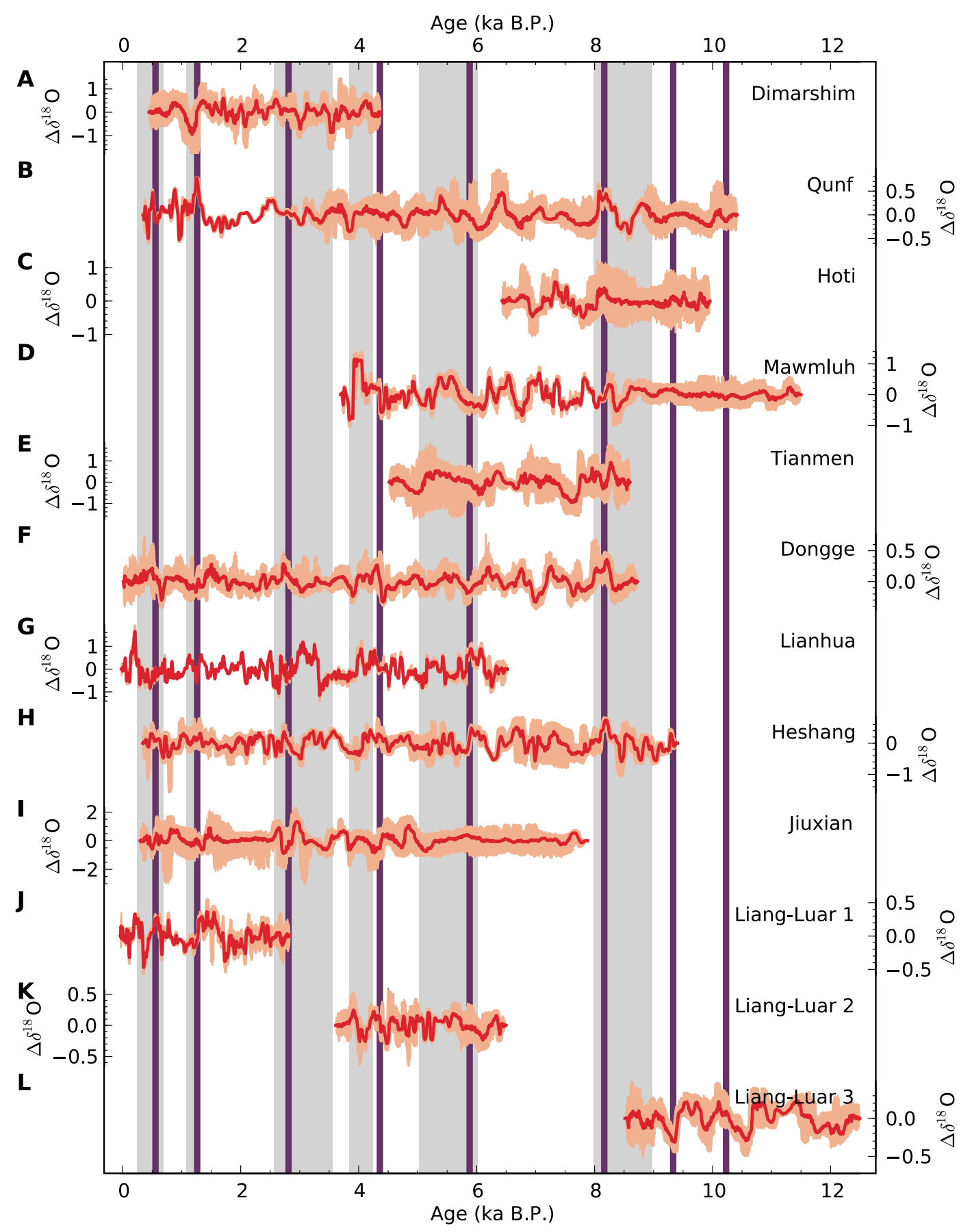

Fig. S1. Residual oxygen isotope records $\Delta \delta^{18} \mathrm{O}$ analysed in this study (all measured in units of [\%०VPDB]). Median (thick red lines) and 90\% confidence intervals (orange shading) from an ensemble of 100 time series realisations of the COPRA algorithm for transferring dating uncertainties to uncertainties in proxy values are shown (Breitenbach et al., 2012). Bond events (violet lines) and RCC episodes (grey bars) are displayed for reference. Note that the Liang-Luar record had to be divided into three sections with a temporal resolution that is sufficiently high for applying the COPRA framework. 


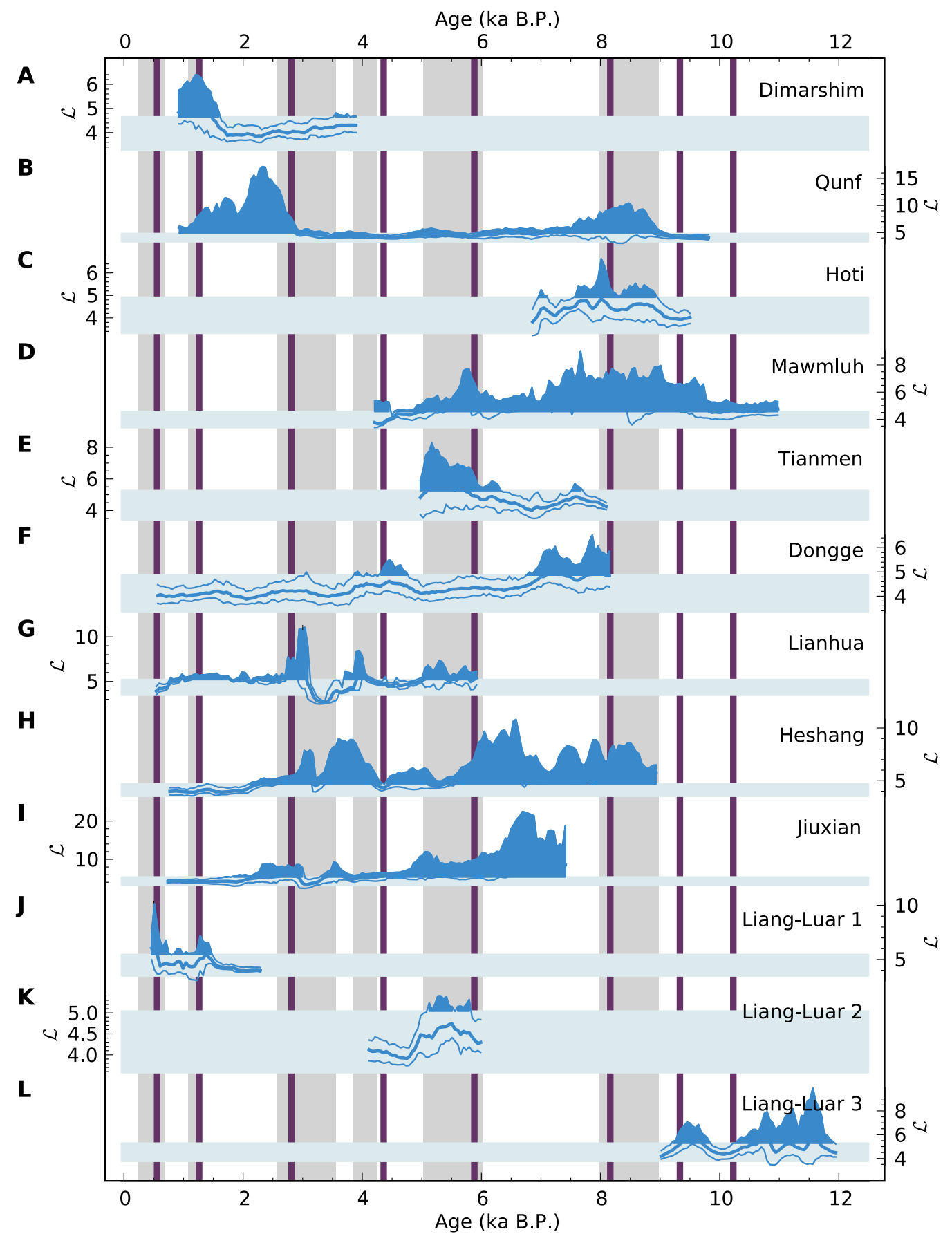

Fig. S2. Time-evolution of an indicator of rapid dynamical change $(\mathrm{RN}$ average path length $\mathcal{L})$ in monsoonal strength fluctuations obtained from a sliding window analysis of the residual oxygen isotope records displayed in Fig. S1. Median (thick dark blue lines) and $90 \%$ confidence intervals (thin dark blue lines) from an ensemble of 100 time series realisations of the COPRA algorithm for transferring dating uncertainties to uncertainties in proxy values are shown. The predominant dynamical regime is marked by $90 \%$ confidence bounds from a stationarity test (horizontal light blue bars, Donges et al. (2011a,b)). Deviations from this regime indicate epochs of significant climatic change (dark blue fillings). Bond events (violet lines) and RCC episodes (grey bars) are displayed for reference. 


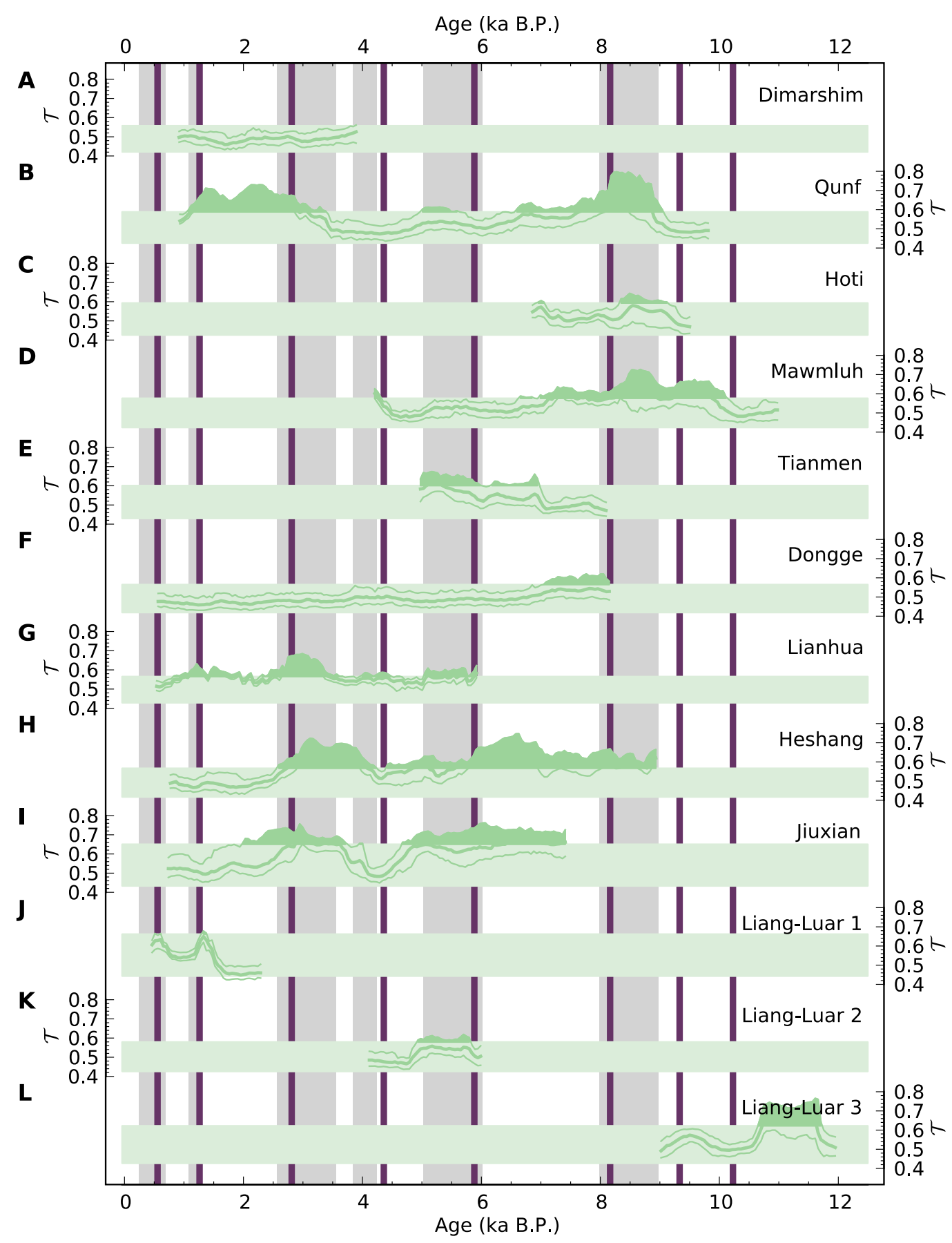

Fig. S3. Time-evolution of the regularity ( $\mathrm{RN}$ transitivity $\mathcal{T}$ ) of variations in monsoonal strength obtained as in Fig. S2 (colour coding is analogous to Fig. S2 after replacing blue by green shades). Here, deviations from the predominant dynamical regime indicate epochs of significantly enhanced or diminished regularity of monsoonal variability (dark green fillings). 